\title{
Enfermedad de Weber-Christian. A propósito de un caso
}

\author{
Weber-Christian Disease. A case report
}

\author{
Cátia Ribeiro Santos, Ana Luisa Albuquerque, Juliana Silva, Helena Carrondo \\ Serviço de Medicina II, Centro Hospitalar de Leiria, Portugal
}

\begin{abstract}
Weber-Christian disease is a skin condition that features recurring inflammation in the subcutaneous fat layer and systemic symptoms. The disease is a diagnosis of exclusion characterized by a lobular panniculitis without vasculitis in histopathology.

We report an 80-year-old man with constitutional symptoms and relapsing nonsuppurative nodular panniculitis.

The purpose of the case described is to emphasize that rare diseases may occur, therefore their diagnosis can only be made with previous medical knowledge and the delay, or even the absence, in the diagnosis of these pathologies could interfere with the quality of life of patients.

Palabras clave: enfermedad de Weber-Christian, paniculitis idiopática lobular, tratamiento corticoideo.

Keywords: Weber-Christian disease, idiopatic lobular panniculitis, corticosteroid therapy.
\end{abstract}

\section{INTRODUCTION}

Panniculitis refers to a broad spectrum of diseases that involve inflammation of the subcutaneous fat layer of the skin'. WeberChristian disease is an eponym for a form of panniculitis called idiopathic nodular panniculitis and the first cases as a distinct entity were described at the end of the 19th century and the beginning of the 20th century². This disease is characterized by recurrent subcutaneous inflammatory painful nodules, usually located in limbs and torso; systemic symptoms and signs such as relapsing fever episodes, fatigue and polyarthralgia are also frequent and may involve the lungs, heart, intestines, spleen, kidney, adrenal glands, and even orbits ${ }^{3}$.

Weber-Christian disease can be described as an autoinflammatory disease affecting adipose tissue and its aetiology is unknown. The key pathologic finding on histological examination is a nodular inflammatory pattern of the fat lobules. When no other cause of lobular panniculitis can be identified (such as systemic lupus, factitious, pancreatic associated, histiocytic cytophagic and alpha1-antitrypsin deficiency panniculitis) Weber-Christian disease can be diagnosed by exclusion ${ }^{4,5}$.

It has been reported most frequently in people between fourth to seventh decades of life, and $75 \%$ of cases occur in women after the second decade of life. Also, it is an extremely rare condition in children ${ }^{6}$.

The medical community still deliberate whether Weber-Christian disease occurs as a unique disease entity or if it is just a substitute for any lobular panniculitis with unknown cause?

\section{CASE REPORT}

We report the case of an 80-year-old Caucasian male, resident in a rehabilitation care facility, with a past medical history of hypertension and vascular dementia. The patient was admitted to the hospital with a two-month history of relapsing fever episodes associated with generalized weakness and recurrent appearance of multiple painful nonsuppurative swellings on the trunk and thighs. Fever had a daily pattern with peaks of $38-39^{\circ} \mathrm{C}$ on regular antipyretic drugs. There were no reported changes in the patient's medications nor history of thermal, mechanical or chemical trauma. He had recent travel history and local epidemiologic data were unremarkable. The patient received prior antibiotic therapy with no clinical improvement.

On the physical exam the patient was conscious, alert, pallor, no jaundice and no rash. His vitals were: blood pressure $123 / 69 \mathrm{mmHg}$, regular heart rate at $95 \mathrm{bpm}$, regular respiratory rate at $12 \mathrm{cycle} / \mathrm{minute}$, temperature $38.2^{\circ} \mathrm{C}$, oxygen saturation $96 \%$ on room air. Chest wall and abdominal examination reveals erythematous, oedematous and tender subcutaneous nodules well circumscribed in the right chest wall as well as in anterior abdominal wall (Figure 1), without fluctuation. There was a depressed and atrophied scar of old incisions over right thigh. Other systemic examinations were unremarkable.

Laboratory investigations showed raised inflammatory markers (C-reactive protein (CRP) $59.3[<0.5 \mathrm{mg} / \mathrm{L}]$, leucocytes $10.300 / \mathrm{mL}$, neutrophils $7000 / \mathrm{mL}$, lymphocytes $2100 / \mathrm{mL}$, elevated erythrocyte sedimentation rate (ESR $68 \mathrm{~mm} / \mathrm{h}$ ) and normocytic normochromic anemia (haemoglobin $10.4 \mathrm{~g} / \mathrm{dL}$,

Figure 1. Subcutaneous nodules in the right chest wall ( $A$ and $C$ ) as well as in anterior abdominal wall (B).

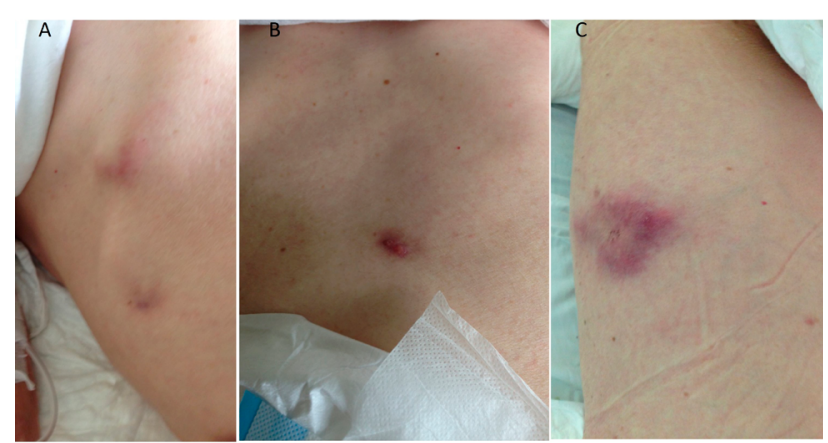


MCV $80.3 \mathrm{fL}$ ) with normal differential count on peripheral smear; renal function, liver biochemistry, lactate dehydrogenase, creatin-phosphokinase, pancreatic amylase were normal. Also, values for ANA, ANCA, anti-ds-DNA, serum protein electrophoresis, complement factors C3 and C4, anti-CCP, and anti-MCV were within the normal range or not detectable, respectively. Normal immunoglobulin assay including IgM 62 mg/dL (normal range, 40-230 mg/dl), IgA 372 mg/ dl (normal range, 22-159 mg/dl), lgG 1079 mg/dl (normal range, 441-1135 mg/dl) and normal serum angiotensin converting enzyme (SACE) activity. Urine analysis with microscopy and urine culture were normal; three repeated sets of blood cultures were negative. Cultures for bacteria, mycobacteria, and fungi from the swelling in right thigh revealed no growth. The serologies for the following diseases were negative: Toxoplasmosis, human immunodeficiency virus, Epstein-Barr virus, hepatitis viruses, cytomegalovirus, syphilis, $Q$ fever, brucellosis, and leptospirosis.

Posteroanterior chest radiography, transthoracic echocardiogram, sonography abdomen and computed-tomography whole-body scanning with no abnormal imaging findings.

One of the painful nodules was removed and histopathologic findings revealed neutrophilic panniculitis with basophilic degeneration of collagen with haemorrhage and necrosis of subcutaneous tissue, inflammatory cellular infiltrate which was mainly composed lymphocytes infiltrating all layers of skin and band of fibroblasts and inflammatory cells in bloody stroma with no vasculitis or malignant cells (Figure 2).

Based on this clinical findings and results of the diagnostic workup, the final diagnosis of a Weber-Christian disease was established. Following treatment with high dose prednisolone, the patient's condition improved significantly and temperature as well as inflammatory markers returned back to normal levels rapidly. Also, the nodules clearly reduced in size, and the patient only reported minor pain on pressure. Therefore, patient was discharged from the hospital and on a routine check-up in the following month he presented in very good general condition with no more signs of inflammation or relapsing nodules.

\section{DISCUSSION}

The term "panniculitis" refers to a group of inflammatory disorders in which the primary site of inflammation is the subcutaneous fat ${ }^{8}$. The diagnosis can be challenging not only because different forms of panniculitis may be present with similar clinical findings, but also many of those are rare ${ }^{5,9}$. Weber-Christian disease is called idiopathic lobular panniculitis because its aetiology is unknown. Indeed, it is a rare condition characterized by an autoinflammatory disease involving adipose tissue, commonly focusing on subcutaneous nodules, inflammatory cells in the fat lobules, and systemic symptoms, such as the presence of relapsing fever ${ }^{4}$.
Figure 2. Histopathology section of skin biopsy stained with haematoxylin and eosin showing inflammatory cellular infiltrate which is mainly lymphocytes infiltrating all layers of skin and band of fibroblasts and inflammatory cells in bloody stroma.

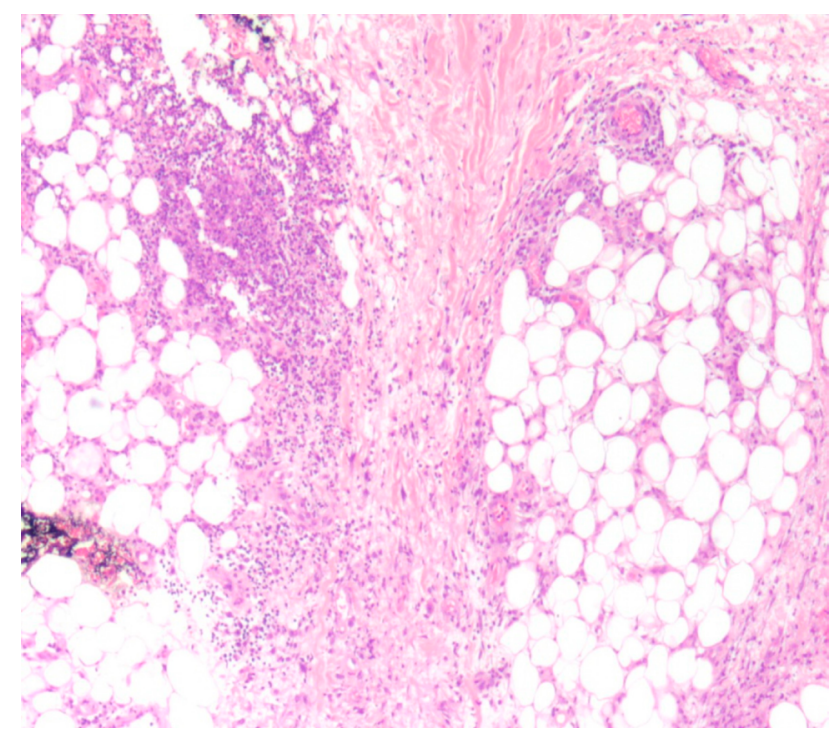

Although the pathogenesis of Weber-Christian disease is not finally resolved, elevated levels of circulating immune complexes in some of the patients may suggest an immunologically mediated reaction ${ }^{11}$.

However, due to the rarity of this condition it is still under debate, if Weber-Christian disease is a unique disease entity, or if it is just a substitute for every panniculitis that does not fit a common diagnosis?. Therefore, increasing study and diagnostic sophistication have differentiated Weber-Christian disease from diseases such as lupus panniculitis, factitial panniculitis, panniculitis associated with pancreatic disease, histiocytic cytophagic panniculitis, and alpha1-antitrypsin deficiency panniculitis ${ }^{10}$. Hence, at this time, the eponym WeberChristian disease still refers to cases of nodular panniculitis with systemic signs and symptoms that remain idiopathic.

The diagnosis of Weber-Christian disease is an exclusion diagnosis ${ }^{5,7}$ with a lobular panniculitis without vasculitis in histopathology as it was demonstrated in our case. In patients with primarily cutaneous manifestations, the clinical course may be characterized by exacerbations and remissions of the cutaneous lesions with minimal systemic complaints for several years before the disorder subsides ${ }^{13}$. On the other hand, this entity has also been described in association with systemic involvement, usually involving liver, spleen, bone marrow and mesenteric adipose tissue ${ }^{12}$.

Skin biopsy is necessary to confirm the diagnosis of panniculitis. Indeed, biopsies can provide valuable information and are indicated both in cases in which the diagnosis is uncertain or in cases in which the clinical course of a panniculitis does not proceed as expected ${ }^{9}$. However, the histopathologic findings are often ambiguous. In many cases, clinical correlation and careful consideration of the differential diagnosis are required to arrive at a correct diagnosis? 
In the present case report, the described symptoms together with lobular panniculitis can also occur in other diseases such as infections, certain malignancies, alpha-1-antitrypsin deficiency, pancreatitis, systemic lupus erythematosus and cytophagic histiocytic panniculitis, rendering Weber-Christian disease an exclusion diagnosis following careful evaluation of the patient. However, all of these diseases, except alpha-1-antitrypsin deficiency, were excluded in our patient by extensive diagnostic evaluation favouring the diagnosis of a Weber-Christian disease. It did not explicitly exclude alpha1-antitrypsin deficiency, because panniculitis is only observed in patients with severe forms of the disease ${ }^{8}$, which would include other symptoms, like COPD and/or liver cirrhosis.

Several treatment options were reported in the treatment of Weber-Christian disease, even though, no specific uniformly effective therapy was recognized. Several reported cases showed patient clinical improvement with the use of the following therapies: fibrinolytic agents, hydroxychloroquine, azathioprine, thalidomide, cyclophosphamide, tetracycline, mycophenolate, and clofazimine ${ }^{11,13}$. Systemic steroids (eg, prednisone) may be effective in suppressing acute exacerbations $^{14}$; and nonsteroidal anti-inflammatory agents (eg, indomethacin) may reduce fever, arthralgias, and other signs of malaise. Involvement of specific organs may require specific supportive drugs ${ }^{12}$. Our patient improved rapidly following corticosteroid treatment. The prognosis of Weber-Christian disease depends on which organs are affected, the severity of organ involvement, and the response to the therapy ${ }^{13}$. Significant morbidity and mortality may occur in patients with inflammation involving visceral organs ${ }^{12}$.

Acknowledgements: The authors thank Dra. Maria Fernanda Silva e Cunha (Pathology department, Centro Hospitalar de Leiria) the great availability.

\section{REFERENCES}

1. Diaz Cascajo C, Borghi S, Weyers W. Panniculitis: definition of terms and diagnostic strategy. Am J Dermatopathol. 2000;22(6):530-549.

2. Steffen $\mathrm{C}$. The men behind the eponym: Frederick Parkes Weber and Henry a. Christian: Weber-Christian disease. Am J Dermatopathol. 2002;24(6):514-517.

3. Haubrich WS. Weber and Christian of Weber-Christian disease. Gastroenterology. 2008;134(4):912.

4. Sharma AK, Sharma PR. Idiopathic lobular panniculitis (Weber Christian disease): a case report. Kathmandu Univ Med J (KUMJ). 2006;4(2):243-245.

5. Oliveira A, Rodrigues S, Jorge R, Crespo J. Doença de Weber-Christian: paniculite sistémica de etiologia desconhecida [Weber-Christian disease: unknown etiology systemic panniculitis]. Acta Med Port. 2010;23(6):1113-1118.

6. Panush RS, Yonker RA, Dlesk A, Longley S, Caldwell JR. Weber-Christian disease. Analysis of 15 cases and review of the literature. Medicine (Baltimore). 1985;64(3):181-191.

7. White JW Jr, Winkelmann RK. Weber-Christian panniculitis: a review of 30 cases with this diagnosis. J Am Acad Dermatol. 1998;39(1):56-62.

8. Requena L, Sánchez Yus E. Panniculitis. Part II. Mostly lobular panniculitis. J Am Acad Dermatol. 2001;45(3):325-364.

9. Patterson JW. Panniculitis. In: Dermatology, 2nd ed, Bolognia JL, Jorizzo JL, Rapini RP (Eds), Elsevier Ltd, London. 2008. p.1515.

10. Geraminejad P, DeBloom JR 2nd, Walling HW, Sontheimer RD, VanBeek M. Alpha-1-antitrypsin associated panniculitis: the MS variant. J Am Acad Dermatol. 2004;51(4):645-655

11. Pongratz G, Ehrenstein B, Hartung W, Schölmerich J, Fleck M. A patient with Pfeifer-Weber-Christian disease--successful therapy with cyclosporin A: case report. BMC Musculoskelet Disord. 2010;11:18.

12. Miranda-Bautista J, Fernández-Simón A, Pérez-Sánchez I, Menchén L. WeberChristian disease with ileocolonic involvement successfully treated with infliximab. World J Gastroenterol. 2015;21(17):5417-5420.

13. Mavrikakis I, Georgiadis T, Fragiadaki K, Sfikakis PP. Orbital lobular panniculitis in Weber-Christian disease: sustained response to anti-TNF treatment and review of the literature. Surv Ophthalmol. 2010;55(6):584-589.

14. Wang Y, Zhao J, Ji LL, Zhang S, Zhang Z. Weber-Christian disease presenting with lung nodules dramatically improved with corticosteroid therapy: one case report and literature review. Int J Rheum Dis. 2018;21(2):573-578. 